\title{
Pressure distribution along the AGS vacuum chambers with new types of pump out conduits
}

\author{
S. Nayak, M. Mapes, L. Smart, D. Weiss
}

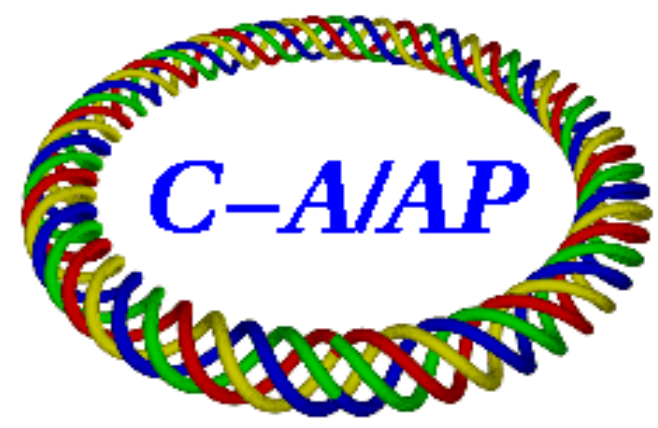

\section{Collider-Accelerator Department Brookhaven National Laboratory Upton, NY 11973}

\section{U.S. Department of Energy}

Office of Science, Office of Nuclear Physics

Notice: This document has been authorized by employees of Brookhaven Science Associates, LLC under Contract No. DE-SC0012704 with the U.S. Department of Energy. The United States Government retains a nonexclusive, paid-up, irrevocable, world-wide license to publish or reproduce the published form of this document, or allow others to do so, for United States Government purposes. 


\title{
Pressure Distribution along the AGS Vacuum Chambers with New Types of Pump out Conduits
}

\author{
S. Nayak, M. Mapes, L. Smart, D. Weiss \\ Brookhaven National Lab, Upton, NY, USA
}

October 28, 2015

(original report issued July 9, 2008)

\begin{abstract}
The AGS HEBT and ring vacuum system is monitored by the discharge current of the magnet ion pumps, which is proportional to the pressure at the inlet port of these ion pumps. The discharge current is measured and suitably calibrated to indicate the ion pump pressure. In order to calculate the vacuum chamber pressure from the ion pump pressure, a detailed analysis is essential to compute their difference in different scenarios. Such analysis has been carried out numerically in the past for the system with the older type of pump out conduits and similar analysis using FEM in ANSYS is presented in this paper with the newer type of pump out conduit.
\end{abstract}

\section{Introduction}

The AGS HEBT and ring vacuum sectors are evacuated and maintained by the magnet ion pumps. There are about 260 standard ion pumps and odd ion pumps around the ring and the HEBTs grouped into 24 ring sectors and 4 HEBT sectors. Each sector contains 10 ion pumps located in an interval of $330 \mathrm{~cm}$ distance. These pumps are connected to the vacuum chambers through a $94 \mathrm{~cm}$ (37") length of pump out conduit [fig-3]. These are the new conduits replaced the old conduits [fig-2] to improve the conductance of the conduit and hence to minimize the difference in the pressure at the vacuum chamber and at the ion pump. The discharge current of these ion pumps which is proportional to the pressure at inlet port of the pumps is used to monitor the pressure of the vacuum system. Considerable pressure gradient exists between the ion pump and the vacuum chamber due to the conductance limitation of the pump elbow and the vacuum chamber itself. The magnitude of the pressure gradient depends upon the conductance of the system, outgassing rate, leak rate, the pump capacity and the distance between the pumps. The analysis is carried out for a standard pump [fig-6] we are using now and two types of pump out conduits [fig-3] using finite element method (FEM). The FEA is executed in ANSYS after converting a vacuum problem in to a thermal problem with proper change in input parameters [2]. The previous technical note [1] is based on the numerical method with few simplifications. The FEM approach without any simplifications is expected to produce accurate results.

Fig-1 and fig-4 show the half symmetry of a HEBT sector and symmetric model used for analysis when $\mathrm{L}=330 \mathrm{~cm}$. The effective gap between the operating pumps becomes $\mathrm{L}=660 \mathrm{~cm}$ and $\mathrm{L}=1650 \mathrm{~cm}$ when $50 \%$ and $20 \%$ ion pumps operate respectively. Symbols used in this report are as follows. 
$\mathrm{P}_{\mathrm{c}}=$ Chamber pressure

$\mathrm{P}_{\mathrm{i}}=$ Ion pump pressure

$\mathrm{Q}=$ Gas flow (torr. $1 / \mathrm{s}$ )

$\dot{q}=$ Volumetric heat generation $\left(\mathrm{W} / \mathrm{cm}^{3}\right)$

$q=$ Outgassing rate $\left(\right.$ Torr $\left.-1 / \mathrm{s}-\mathrm{cm}^{2}\right)$

$\mathrm{C}=$ Gas conductance $(1 / \mathrm{s})$

$\mathrm{K}=$ Material thermal conductivity

$P=$ Perimeter of the vacuum chamber

$\mathrm{L}=$ Ion pump separation

$1=$ Length of the vacuum chamber

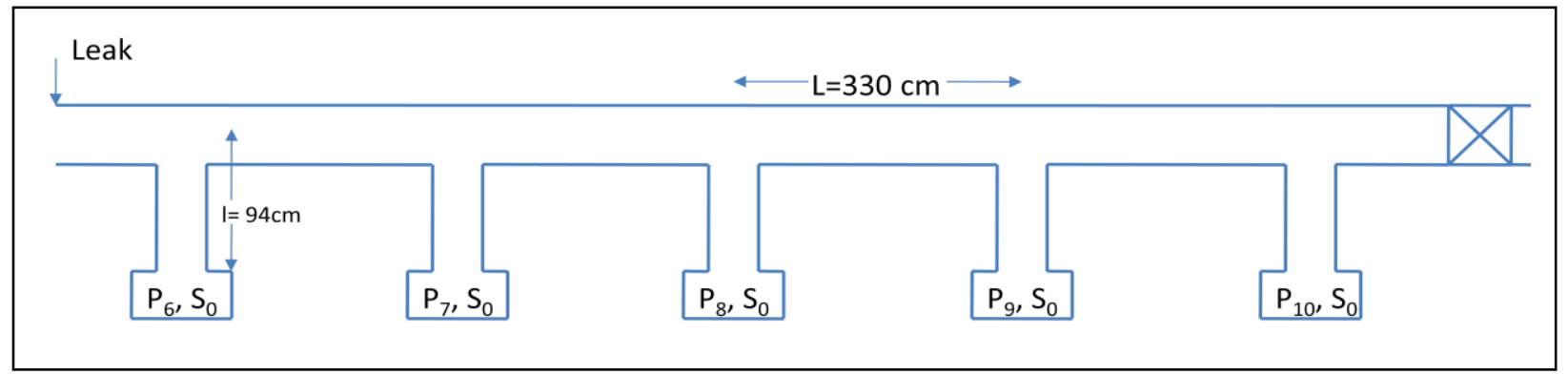

Fig-1: Half symmetric view of a HEBT sector
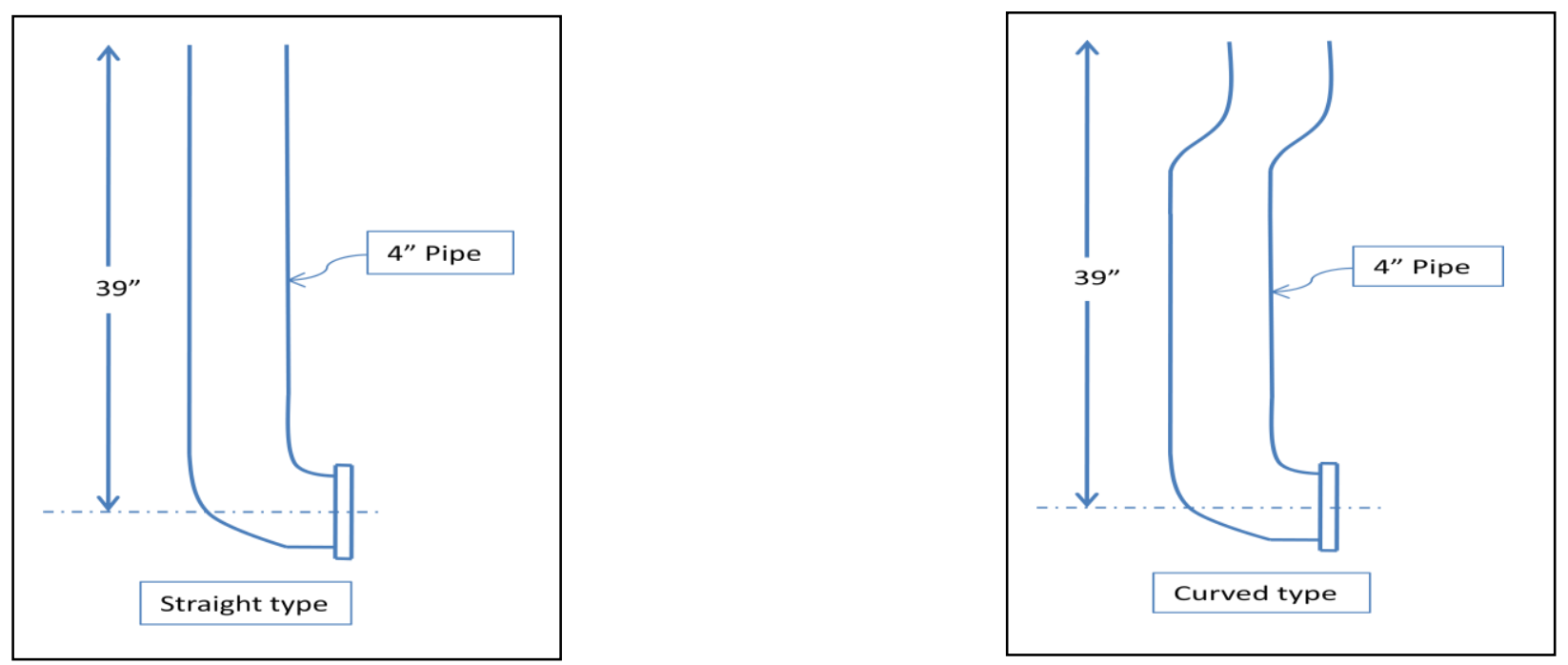

Fig-2: Types of pump out conduit used earlier in AGS HEBT ring sector [4] 

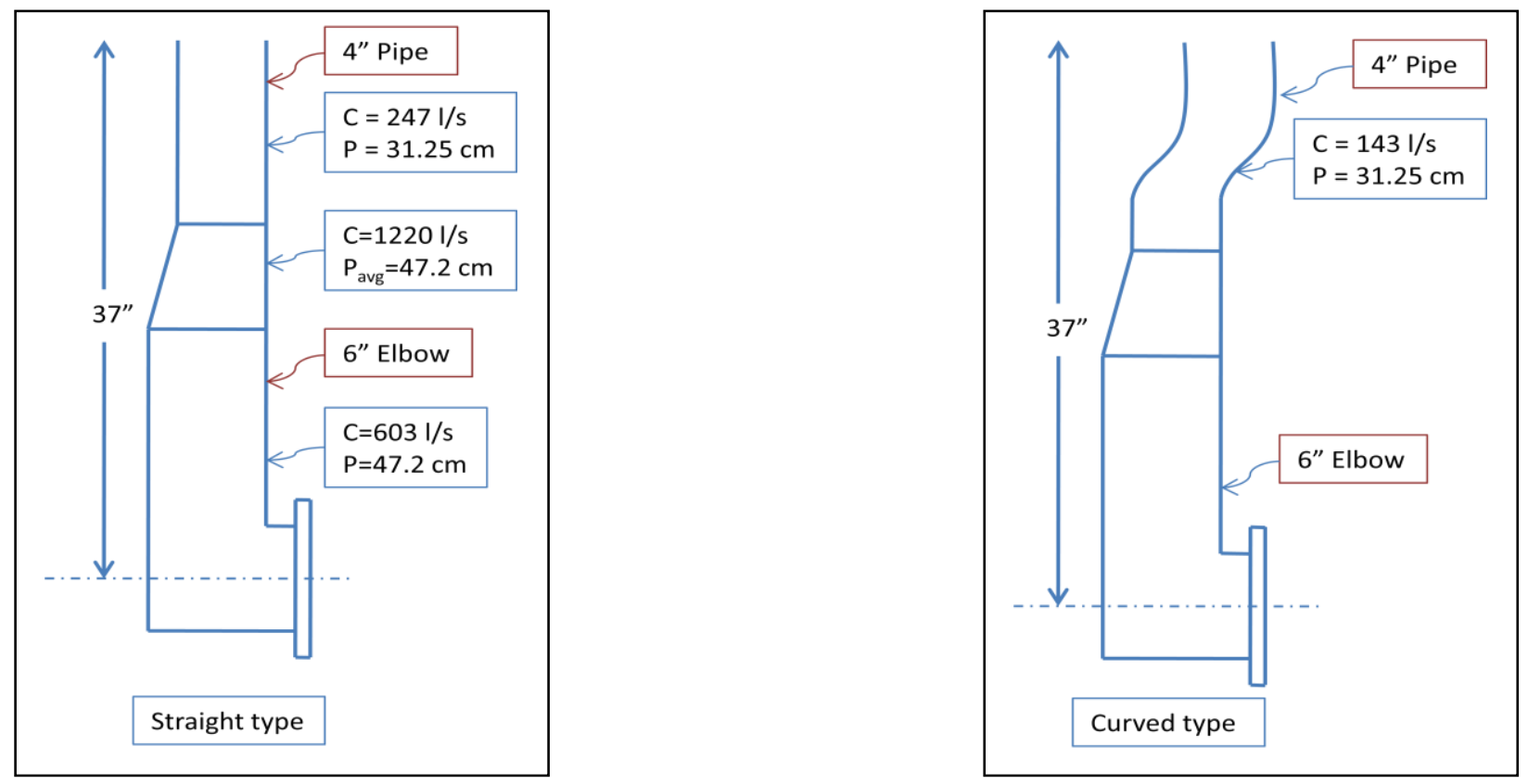

Fig-3: Types of pump out conduit used now in the AGS HEBT ring sector [3]

\section{Finite element modeling}

The FEM has proven to be a useful tool for designing complex and long vacuum systems. For this one can develop finite element programs or use commercially available FEM package (like ANSYS) with heat transfer capabilities. The dimensional analogy existing between one-dimensional thermal conduction and gas conduction [table-1] has been utilized to calculate pressure distribution in the AGS HEBT vacuum systems. In this approach the pressure, gas conduction, gas load and pumps are equivalent to temperature, thermal conduction, the heat generation and the heat sink respectively.

\begin{tabular}{|c|c|c|c|}
\hline \multicolumn{2}{|l|}{ Vacuum } & \multicolumn{2}{|l|}{ Thermal } \\
\hline Quantity & Unit & Quantity & Unit \\
\hline $\mathrm{P}$ - Pressure & Torr & T - Temperature & ${ }^{\circ} \mathrm{C}$ \\
\hline C-Gas conductance & $\mathrm{L} / \mathrm{s}$ & KA/L-Thermal Conductance & $\mathrm{W} /{ }^{\circ} \mathrm{C}$ \\
\hline$q$-Gas source & Torr-L/s-cm ${ }^{2}$ & $\dot{q}$-Heat generation & $\mathrm{W} / \mathrm{cm}^{3}$ \\
\hline
\end{tabular}

Table-1: Vacuum-thermal analogy

\subsection{Element}

For one-dimensional heat conduction analysis two node conduction element (link-32) is used. It is a uniaxial element with ability to conduct heat between its nodes. The element has a single degree of freedom, temperature, at each node point. Two nodes (define the length of element), a cross-sectional area, and the material properties (thermal conductivity) define the element. Volumetric heat generation rate and temperature boundary condition completely define the model. 


\subsection{Properties}

- Volumetric heat generation is set equal to degassing rate of the vacuum component.

- Cross sectional area of the element is set equal to the surface area per unit length (perimeter) of the vacuum component.

One dimensional heat conduction equation with heat generation can be given by

$$
\begin{aligned}
& K A \frac{d T}{d X}=\dot{q} A l \\
& \Rightarrow \dot{q}=\frac{K}{l} \frac{d T}{d X}-----------------(1)
\end{aligned}
$$

Now, the gas flow rate equation in a vacuum system is

$$
\begin{aligned}
& Q=C \times d p \\
& \Longrightarrow \frac{Q}{P \times d X}=\frac{C}{p} \frac{d p}{d X} \\
& \Longrightarrow q=\frac{C}{p} \frac{d p}{d X}-----1----(2)
\end{aligned}
$$

By applying vacuum-thermal equivalence in equations (1) and (2), we get

$$
\begin{aligned}
& \frac{K}{l}=\frac{C}{P} \\
& \Rightarrow K=\frac{C P}{l}-------------------(3)
\end{aligned}
$$

From equation (3), it is clear that perimeter in a vacuum system will be treated as cross sectional area equivalent (quantitative wise) in thermal analysis.

- Material thermal conductivity is calculated from equation (3) and put in the material properties.

\subsection{Pump}

Heat generation rate of pump element is assumed to be zero. Other parameters are set as follows. Gas conductance $=$ Thermal conductance

$$
\Rightarrow C=\frac{K A}{l}
$$

Thermal conductivity, $\mathrm{K}$ is set equal to the pumping speed and the area of cross section is set to the length of the line representing pump model.

$$
\begin{aligned}
& \therefore K=l \\
& C=A
\end{aligned}
$$

Temperature at the free ends of the pump elements are specified as the pressure that pump can achieve.

It can be noted that pumping speed is a function of the pump pressure [fig-], which is again depends upon the gas load. So depending upon the gas load handled by the pump, pump pressure and pumping speed are selected from fig-6. 
Conductance and perimeter of the pump out conduit are mentioned in fig-3. Linear conductance of the elliptical vacuum chamber is equal to $192001 . \mathrm{cm} / \mathrm{s}$ [1]. At the intersection of the pump out conduit and the vacuum chamber, a pseudo element of conductance $600 \mathrm{l} / \mathrm{s}$ is introduced to account the resistance due to $90^{\circ}$ bend. A finite element model of fig-4 is shown in fig-5.

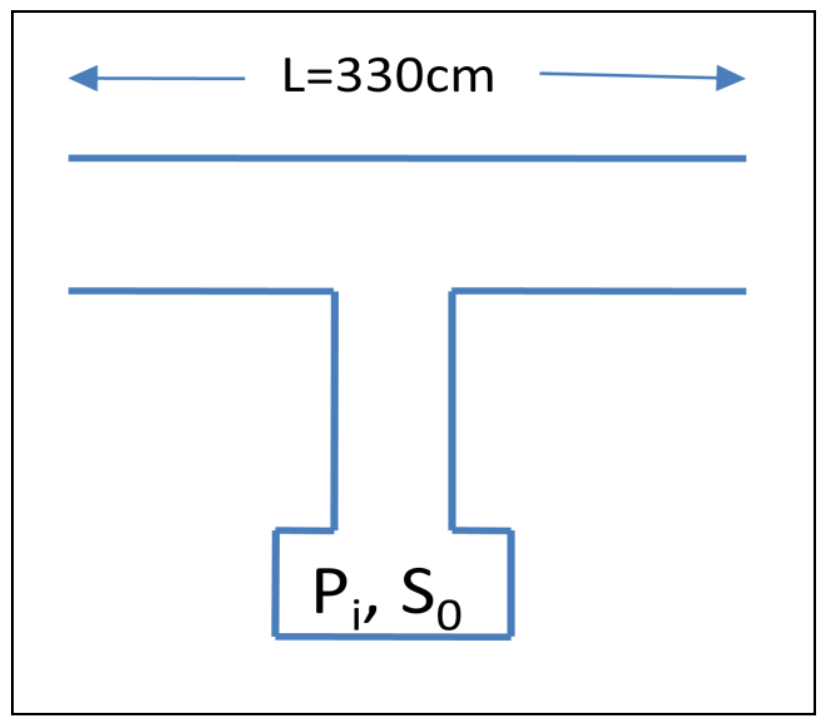

Fig-4: Symmetric model used for $\mathrm{L}=330 \mathrm{~cm}$

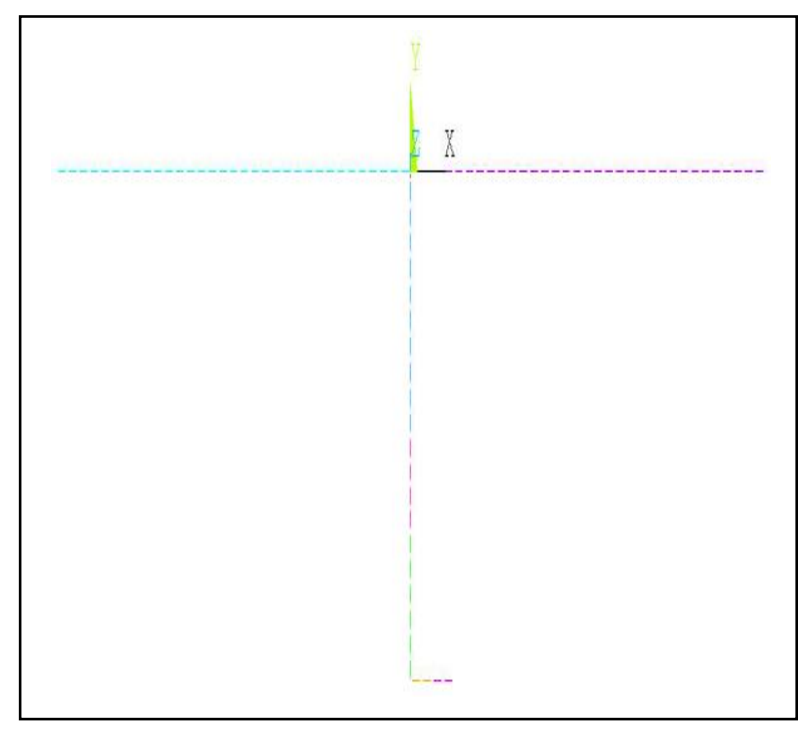

Fig-5: Finite element model $\mathrm{L}=330 \mathrm{~cm}$

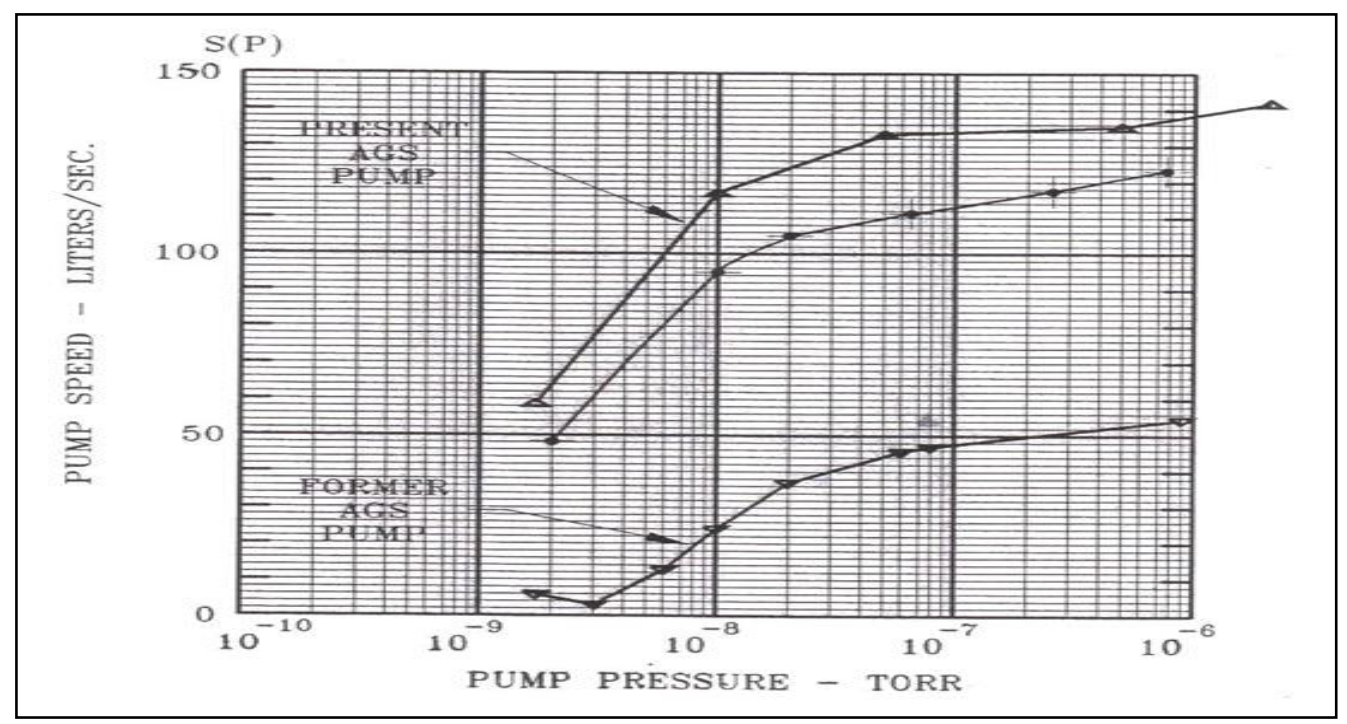

Fig-6: Ion pump speed used in AGS

\section{Pressure Distribution}

In this part, two simplified and most common cases have been considered such as the uniform wall outgassing rate with no leaks and with small leak. Normal operating condition of the ring refers to the outgassing rate of $\sim 5 \times 10^{-11}$ torr. $\frac{l}{\mathrm{~s}} / \mathrm{cm}^{2}$, no leak and with all the pumps are in operation. Nevertheless, the system might operate in worst operating conditions such as high outgassing rate, less 
number of pump in operation and also with leak. In the analysis, different operating conditions are studied and presented.

\subsection{Uniform Outgassing With No Leaks}

Three different outgassing rates such as $1 \times 10^{-10}$ torr. $\frac{l}{\mathrm{~s}} / \mathrm{cm}^{2}, 5 \times 10^{-11}$ torr. $\frac{\mathrm{l}}{\mathrm{s}} / \mathrm{cm}^{2}$ and $1 \times$ $10^{-11}$ torr. $\frac{l}{s} / \mathrm{cm}^{2}$ have been studied with $100 \%(\mathrm{~L}=330 \mathrm{~cm}), 50 \%(\mathrm{~L}=660 \mathrm{~cm})$ and $20 \%(\mathrm{~L}=1650 \mathrm{~cm})$ ion pumps are in operation. Table- 2 shows the pumping speed and the pumping pressure for $\mathrm{L}=330 \mathrm{~cm}$ at different outgassing rate. Fig-7 shows that the average pressure in the ring with all the pumps operating is close to $2.5 \times 10^{-8}$ torr for outgassing rate of $5 \times 10^{-11}$ torr. $\frac{l}{s} / \mathrm{cm}^{2}$ which is expected [1]. Fig- 8 shows the pressure distribution along the vacuum chamber as compared to the ion pump pressure at different outgassing rate for both straight and curve pump out conduit. Under normal operating condition, the chamber pressure can be 3 and 3.42 times the measured pressure for straight and curved pump out conduit respectively. Under similar condition, lower outgassing rate results lower pressure ratio and higher ion pump separation results higher pressure ratio.

\begin{tabular}{|c|c|c|c|}
\hline \multirow{2}{*}{ Quantity } & \multicolumn{3}{|c|}{ Outgassing rate (torr.1/s- $\mathrm{cm}^{2}$ ) } \\
\cline { 2 - 4 } & $1 \mathrm{e}-10$ & $5 \mathrm{e}-11$ & $1 \mathrm{e}-11$ \\
\hline Pumping Speed (1/s) & 121 & 110 & 72 \\
\hline Pump pressure (torr) & $1.5 \mathrm{e}-8$ & $8.27 \mathrm{e}-9$ & $2.52 \mathrm{e}-9$ \\
\hline
\end{tabular}

Table-2: Pumping speed and corresponding pumping pressure for $\mathrm{L}=330 \mathrm{~cm}$

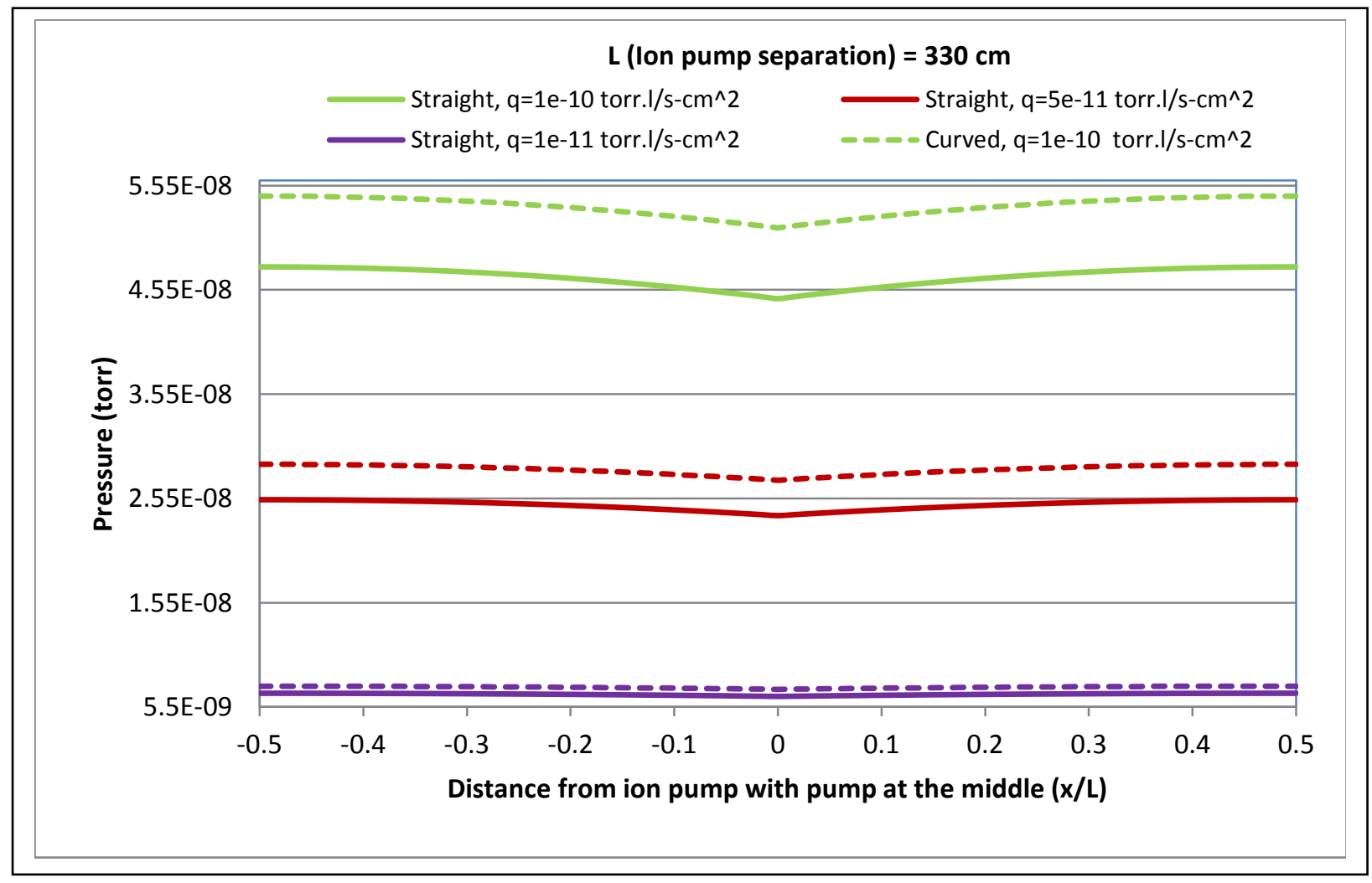

Fig-7: Pressure along the vacuum chamber at different outgassing rate 


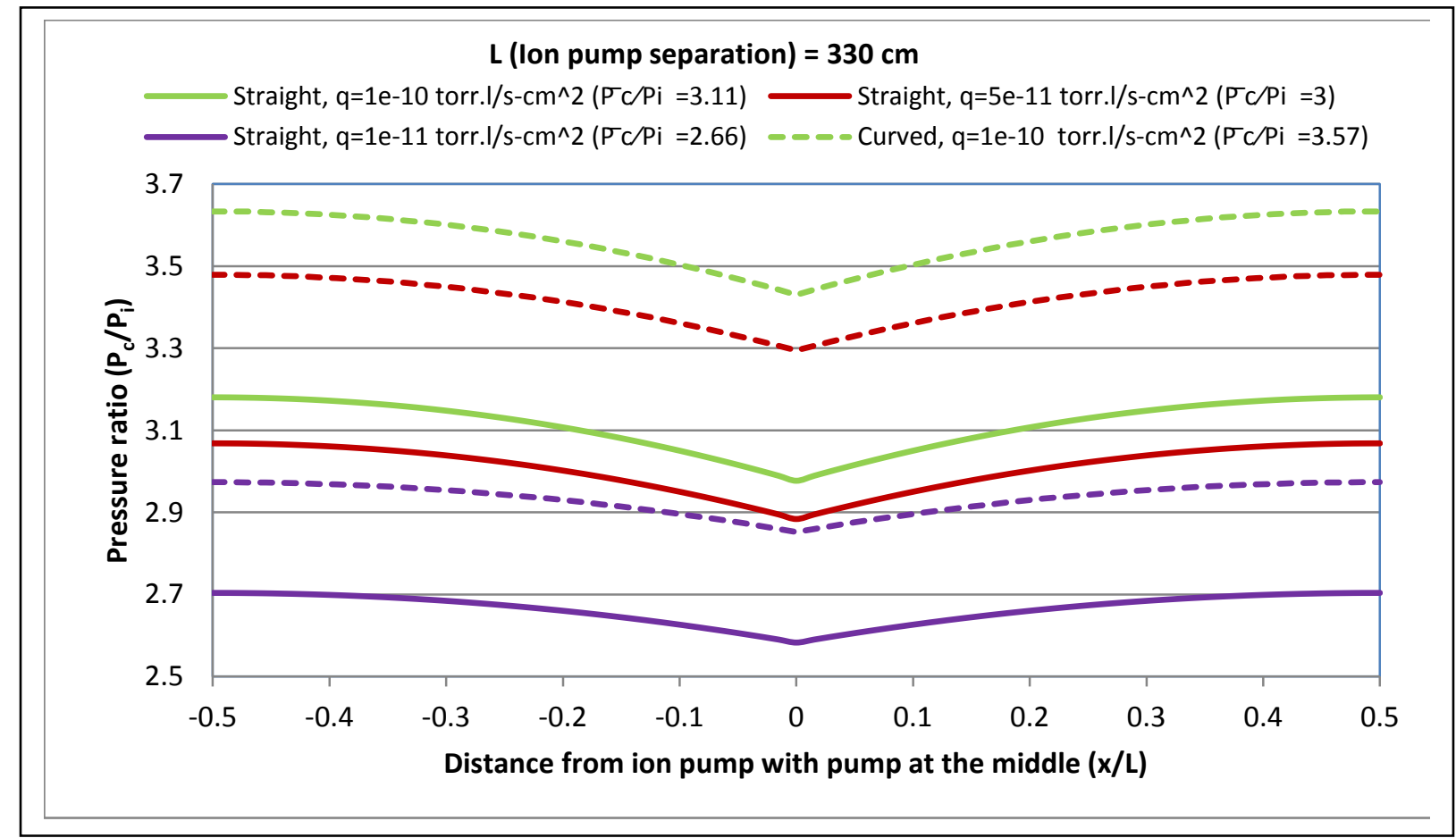

Fig-8: Pressure ratio along the vacuum chamber at different outgassing rate

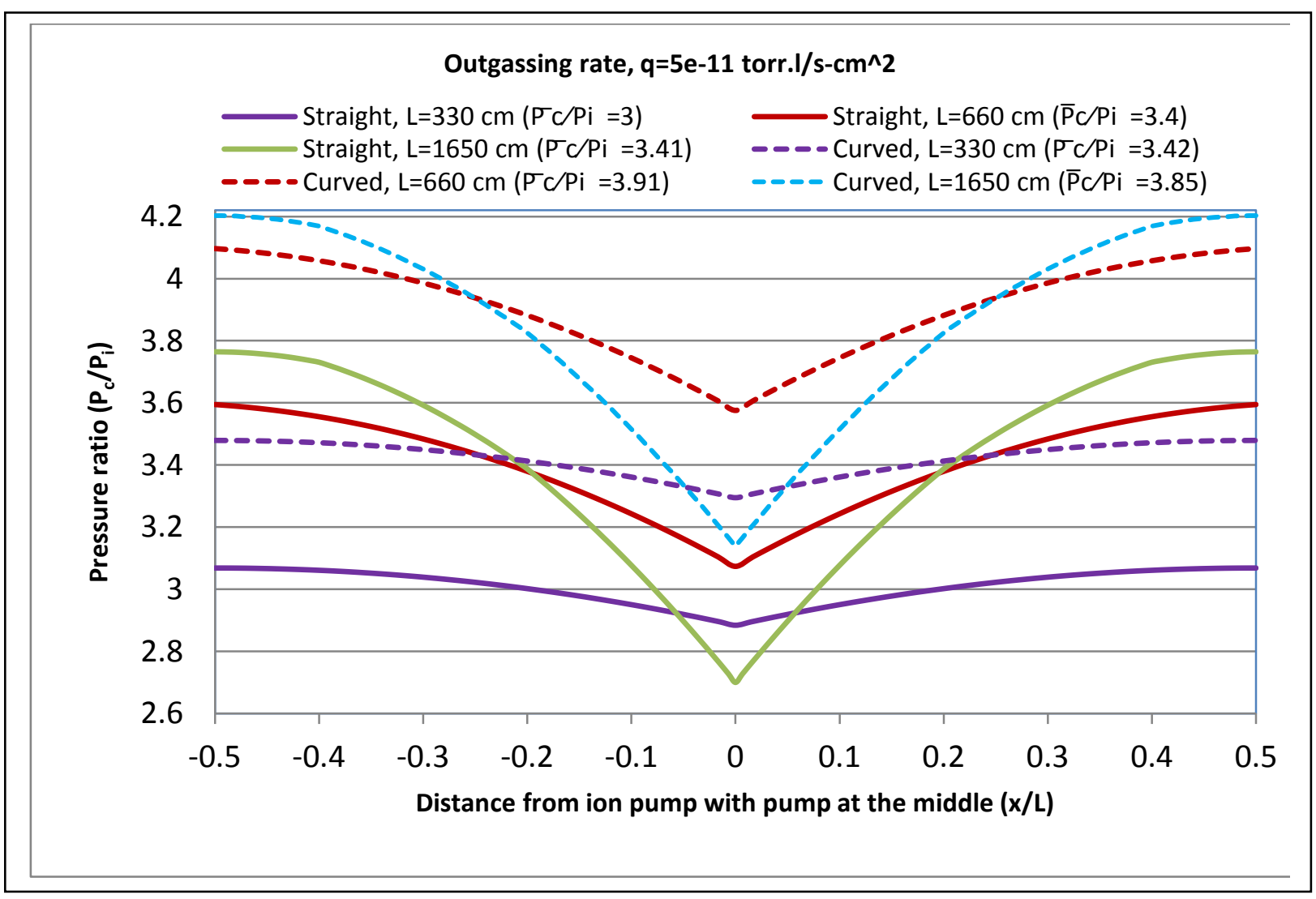

Fig-9: Pressure ratio along the vacuum chamber at different ion pump separation 


\subsection{Uniform Outgassing With Small Leak}

Half symmetric model of one sector [fig-1] with all the pumps in operation is taken for analysis and the gas leak rate of $10^{-5}$ torr.1/s at the midway of the sector is considered. In addition to leak, uniform outgassing rate of $5 \times 10^{-11}$ torr. $\frac{l}{s} / \mathrm{cm}^{2}$ is also applied. It can be assumed without significant error that the gas load due to leak is shared by the pumps in exponentially decreasing order with distance from the location of leak. This assumption helps to define the pumping speed and pressure of the individual pump. Pressure in the vacuum chamber near to leak can go up to $\sim 10^{-7}$ torr [fig-10] and the average pressure ratio in the zone near to the leak increases to 3.17 and 3.56 (with leak) [fig-11] from 3 and 3.42 (without leak) [fig-8] for straight and curved conduits respectively. The pressure ratio decreases with increase in distance from the location of leak. With increase in ion pump separation and outgassing rate, the pressure ratio will increase, however with decrease in out gassing rate pressure ratio won't be affected much as the gas load due to leak will dominate.

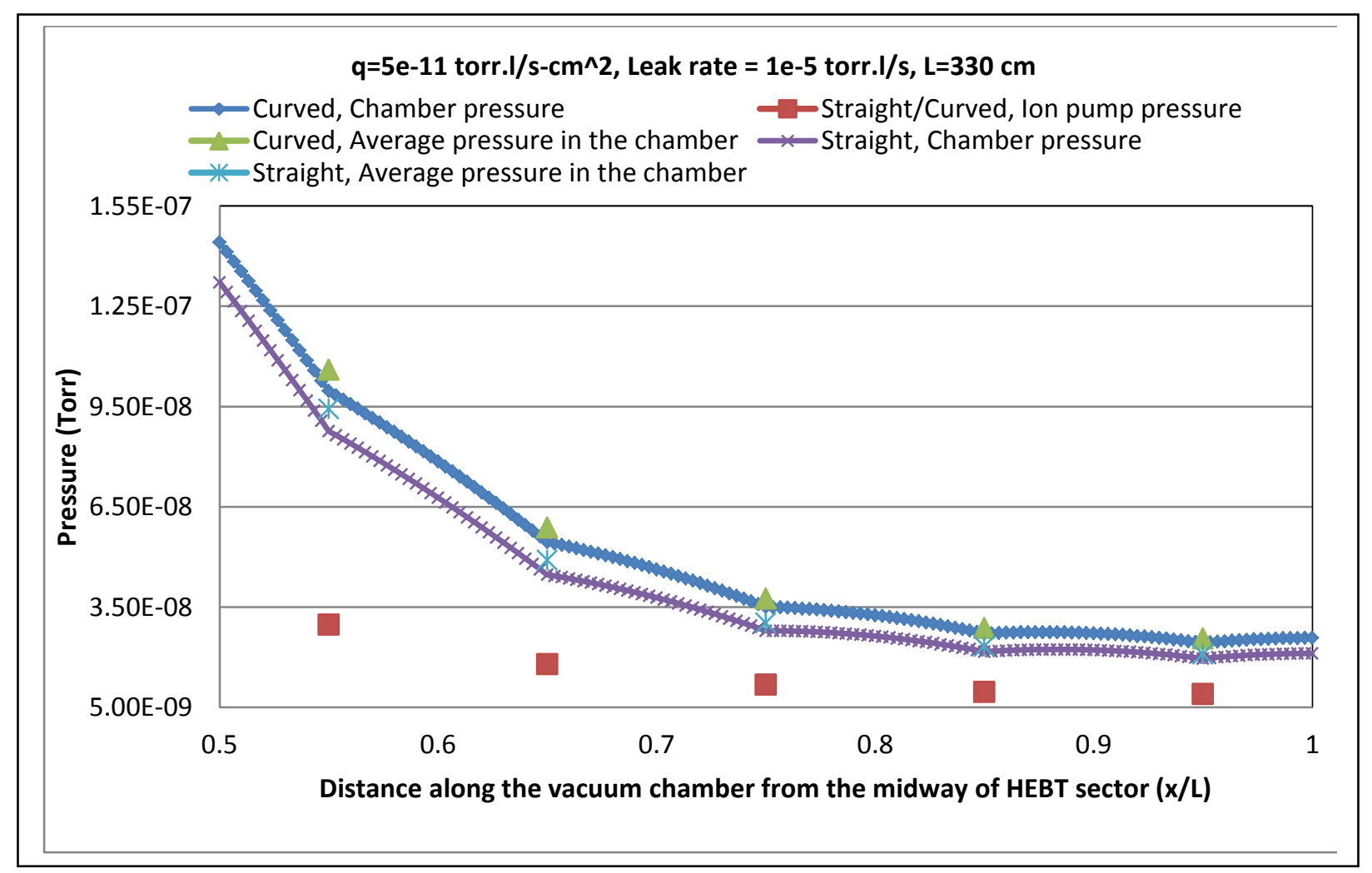

Fig-10: Pressure along the vacuum chamber with leak at the middle of HEBT sector 


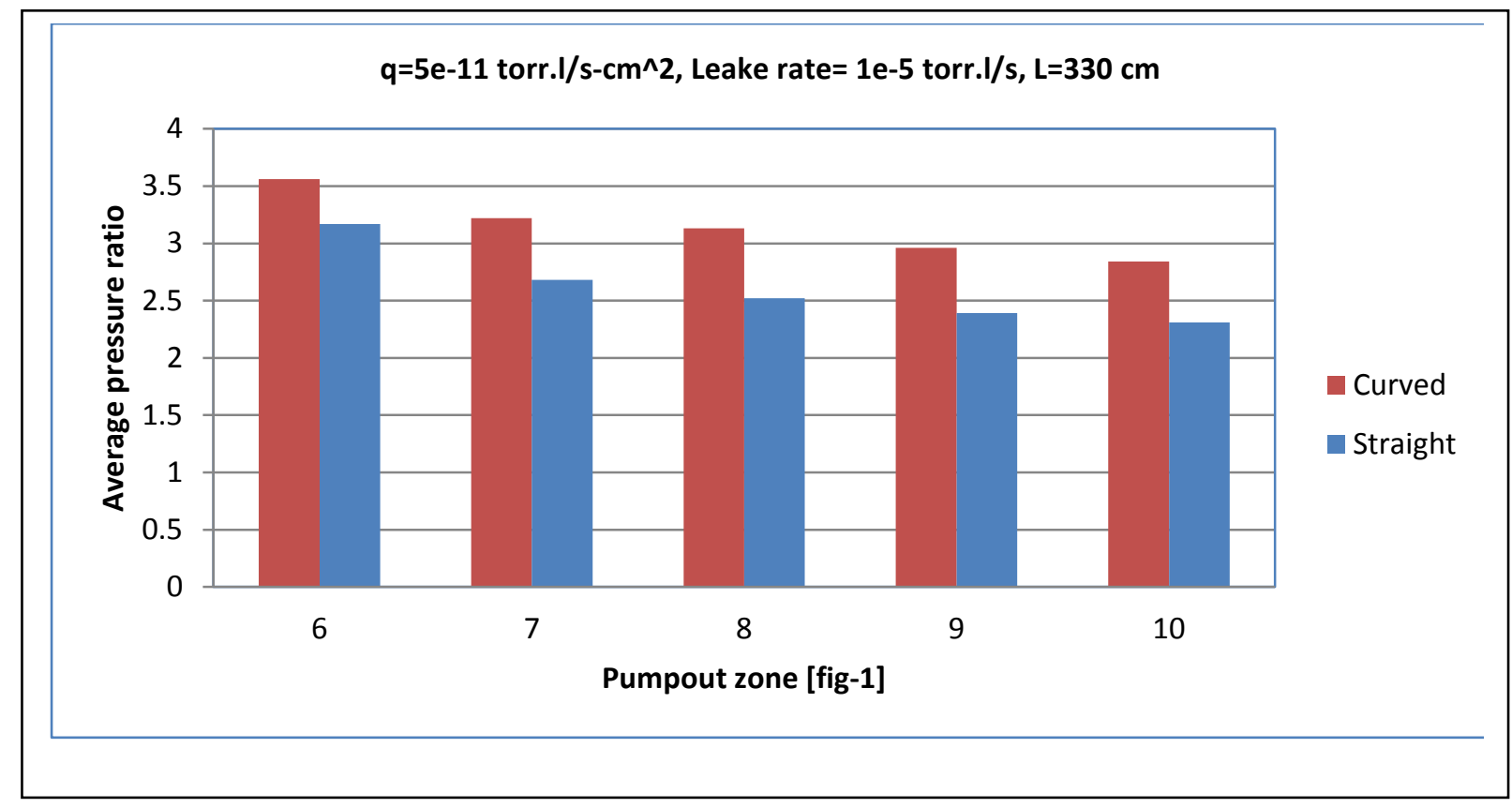

Fig-11: Average pressure ratio at different pump out zone with leak at the middle of HEBT sector

\section{Conclusion}

The pressure distribution along the AGS ring vacuum chamber has been analyzed by FEM (ANSYS) using vacuum thermal analogy for two types of pump out conduit. The analysis has been carried out elaborately for two different cases such as uniform outgassing rates and uniform outgassing rate with small leak. In the first case three different types of operating conditions have been considered such as $20 \%, 50 \%$ and $100 \%$ ion pumps in operation.

For uniform outgassing rate of $5 \mathrm{e}-11$ torr. $1 / \mathrm{s}-\mathrm{cm}^{2}$ and when $100 \%$ pumps are in operation, the ratio between the average chamber pressure and the measured ion pump pressure (pressure ratio) can be 3 and 3.42 for straight and curved type of pump out conduit respectively. In these conditions, chamber pressure lies close to $2.5 \times 10^{-8}$ torr. This ratio increases with outgassing rate and with ion pump distance.

For uniform outgassing rate of $5 \mathrm{e}-11$ torr. $1 / \mathrm{s}-\mathrm{cm}^{2}$ and leak rate of $10^{-5}$ torr.1/s, the ratio between the average chamber pressure and the measured ion pump pressure increases to 3.17 and 3.56 for straight and curved type of pump out conduit respectively. The pressure ratio will increase in ion pump separation and outgassing rate. The chamber near to the location of leak attains pressure in the order of $10^{-7}$ torr.

The previous technical note [1] shows lower pressure ratio even using the pump out conduits with lower conductance. This can be attributed to the over simplification approach used in the numerical analysis and also it might be due to the type of pump used in the analysis about which detailed information are not available. 


\section{References}

1. Pressure Distribution along the AGS Vacuum Chambers. H.C. Hseuh, AGS/AD/Tech. Note No. 273, Brookhaven National Laboratory, Upton, NY, 11973

2. Calculation of Pressure Distribution in Vacuum Systems* Using a Commercial Finite Element Program. J. Howell, B. Wehrle, Argonne National Laboratory 9700 Cass Ave Argonne ,IL 60439. H. Jostlein, Fermi National Accelerator Laboratory, P.O Box 500 Batavia, IL 60510.

3. Drawing number: D05-M-2546 and D05-M-2548

4. Drawing number: D05-M-1933 and D05-M-1934 